BULL. AUSTRAL. MATH. SOC.

$46 A 05,46 A 14,46 A 15$

VOL. 28 (1983), 383-392.

\title{
ON TWO NEW CLASSES OF LOCALLY CONVEX SPACES
}

\author{
KAZUAKI KITAHARA
}

\begin{abstract}
The purpose of this paper is to introduce two new classes of locally convex spaces which contain the classes of semi-Montel and Montel spaces. Further we give some examples and study some properties of these classes. As to permanence properties, these classes have similar properties to semi-Montel and Montel spaces except strict inductive limits and these classes are not always preserved under their completions. We shall call these two classes as B-semi-Montel and B-Montel spaces. A B-semi-Montel space is obtained by replacing the word "bounded" by "strongly bounded" in the definition of a semi-Montel space. If a $\beta$-semiMontel space is infra-barrelled, we call the space $\beta$-Montel.
\end{abstract}

In a locally convex space $E(\tau)$, if each bounded subset is relatively compact, $E(\tau)$ is semi-Montel. If $E(\tau)$ is infra-barrelled and semiMontel, it is Montel. In this paper we weaken the conditions of being (semi-) Montel and introduce two new classes of locally convex spaces.

One contains the class of all Montel spaces and another contains the class of all semi-Montel spaces. We shall call these two classes . B-Montel and $\beta$-semi-Montel spaces. Now we explain what we investigate in each section. In Section 1, we give some notations and definitions of

Received 20 July 1983.

Copyright Clearance Centre, Inc. Serial-fee code: 0004-9727/83 $\$ \mathrm{~A} 2.00+0.00$. 
$B-($ semi-) Montel space. In Section 2 we give some examples of $\beta$-(semi-) Montel spaces which are not (semi-)Montel. In Section 3 we consider some properties of $\beta-($ semi-) Montel spaces. Finally we investigate the separability of a metrizable B-Montel space in Section 4.

\section{Notations and definitions}

Mostly we shall use the notations of [3] and [6]. Let $E(\tau)$ be a Hausdorff topological vector space. Throughout this paper we assume that $E(\tau)$ is a locally convex space over the real or complex field $K$. For the sake of simplicity, it is denoted by lcs $E(\tau)$. $E^{\prime}$ denotes the topological dual space of $E(\tau)$. The dual of $E(\tau)$ always means the topological dual space. When two vector spaces $E$ and $F$ over $K$ form a dual pair, $\sigma(E, F), \tau(E, F), B(E, F)$ and $\beta^{*}(E, F)$ are the topology of uniform convergence on the set of all finite subsets, all absolutely convex $\sigma(F, E)$-compact subsets, all $\sigma(E, E)$-bounded subsets and all $B(E, E)-$ bounded subsets of $E$ on $E$ respectively. Let $E(\tau)$ be a locally convex space and $E^{\prime}$ be its dual. $\tau_{c}\left(E^{\prime}, E\right)$ means the topology of uniform convergence on the set of all $\tau$-precompact subsets of $E$ on $E^{\prime} \cdot E(\tau)$ is said to be a countably barrelled space if each $\sigma\left(E^{\prime}, E\right)$-bounded subset of $E^{\prime}$ which is the countable union of equicontinuous subsets of $E^{\prime}$ is itself equicontinuous [4]. $E(\tau)$ is said to be a $W$-space if each $\sigma\left(E^{\prime}, E\right)$-bounded subset of $E^{\prime}$ is $\beta\left(E^{\prime}, E\right)$-bounded [5]. We say that an lcs $E(\tau)$ possesses a fundamental sequence of bounded subsets if there exists a sequence $B_{1} \subset B_{2} \subset \ldots \subset B_{n} \subset \ldots$ of bounded subsets in $E(\tau)$ such that every bounded subset $B$ is contained in some $B_{k}$.

DEFINITION. Let $E(\tau)$ be a locally convex space and $E^{\prime}$ be its dual.

(1) $E(\tau)$ is said to be a $\beta$-semi-Montel space if each $B\left(E, E^{\prime}\right)-$ bounded subset is relatively $\tau$-compact.

(2) $E(\tau)$ is said to be a $\beta$-Montel space if it is a $\beta$-semi-Montel space and infra-barrelled.

REMARK I. Clearly every Montel space is B-Montel. Every semiMontel space is $\beta$-semi-Montel and every $\beta$-Montel space is $\beta$-semi-Montel.

PROPOSITION 1. Let $E(\tau)$ be a B-semi-Montel space and $E^{\prime}$ be its 
dual. Then the following conditions are equivalent:

(1) $E(\tau)$ is a semi-Montel space;

(2) $E(\tau)$ is a W-space;

(3) $E(\tau)$ is sequentially complete.

Proof. Clearly every semi-Montel space is sequentially complete. If $E(\tau)$ is sequentially complete, $\tau$-boundedness is identical with $\beta\left(E, E^{\prime}\right)$ boundedness. Hence $E(\tau)$ is a $W$-space. Finally if $E(\tau)$ is a $W$-space, it is semi-Montel from the assumption and definition.

\section{Examples of $\beta-($ semi-)Montel spaces}

EXAMPLE 1. Let $E(\tau)$ be an infra-barrelled locally convex space and not barrelled and $E^{\prime}$ be its dual. Then $E^{\prime}\left(\sigma\left(E^{\prime}, E\right)\right)$ is B-semi-Montel but not semi-Montel. If $B$ is any $B\left(E^{\prime}, E\right)$-bounded subset in $E^{\prime}\left(\sigma\left(E^{\prime}, E\right)\right)$, it is relatively $\sigma\left(E^{\prime}, E\right)$-compact since it is a $\tau$-equicontinuous subset. If $E^{\prime}\left(\sigma\left(E^{\prime}, E\right)\right)$ is semi-Montel, $E(\tau)$ is a barrelled space. Therefore $E^{\prime}\left(\sigma\left(E^{\prime}, E\right)\right)$ is not a semi-Montel space.

EXAMPLE 2. Let $T$ be a completely regular Hausdorff space. $C_{s}(T)$ denotes all continuous real valued functions on $T$ with the topology of simple convergence. Then the dual of $C_{s}(T)$ consists of all bounded Radon measures on $T$ with finite support. We denote this dual by $M_{f}(T)$.

$C_{s}(T)$ is always infra-barrelled from corollary 4 of [2]. If $T$ is $[0,1]$ with the usual topology, $C_{s}([0,1])$ is not barrelled from Corollary 13 of $[2]$. Hence in the dual pair $\left(C_{8}([0,1]), M_{f}([0,1])\right)$, $M_{f}([0,1])\left(\sigma\left(M_{f}([0,1]), c_{s}([0,1])\right)\right)$ is $\beta$-semi-Montel but not semiMontel.

EXAMPLE 3. We give another example from sequences spaces. From Example F of [7],

$$
\psi=\left\{x \in K^{N}: x \text { has finitely many non-zero coordinates }\right\},
$$

however $K$ is a real or complex field. For each element $x$ of $\psi$, a norm is given by $\|x\|_{\infty}=\sup _{i \in N}\left|x_{i}\right| \cdot\left(\psi,\|\|_{\infty}\right)$ is a normed space and not 
barrelled since $A=\left\{x: x \in \psi,\left|x_{n}\right| \leq 1 / n, n=1,2, \ldots\right\}$ is a barrel in $\left(\psi,\|\|_{\infty}\right)$ but not a 0-neighbourhood in $\left(\psi,\|\|_{\infty}\right)$. As $\left(\psi,\|\|_{\infty}\right)$ is a dense subspace of $\left(c_{0},\|\|_{\infty}\right)$, the dual of $\left(\psi,\|\|_{\infty}\right)$ is $l^{l}$. In the dual pair $\left(\psi, l^{1}\right)$, we make some remarks about $l^{1}\left(\sigma\left(l^{2}, \psi\right]\right)$.

(1) $l^{1}\left(\sigma\left(Z^{1}, \psi\right)\right)$ is B-semi-Montel but not semi-Montel.

(2) $l^{\perp}\left(\sigma\left(l^{\perp}, \psi\right)\right)$ is metrizable since it has a countable base of 0-nei ghbourhoods .

From (1) and (2), $Z^{1}\left(\sigma\left(Z^{\perp}, \psi\right)\right)$ is a $\beta$-Montel space but not a Montel space.

Next we give a proposition generalizing Example 3 . Before this, we use the following notations.

Let $X$ be a set such that $|X| \geq \kappa_{0}$ and $K$ be a real or complex field. For an arbitrary positive number $p$ with $1 \leq p<\infty$, we put

$$
\begin{aligned}
& Z^{P}(X)=\left\{\left(z_{x}\right)_{x \in X}:\left(z_{x}\right)_{x \in X} \in K^{X},\left(\sum_{x \in X}\left|z_{x}\right|^{p}\right)^{I / p}<\infty\right\}, \\
& Z^{\infty}(X)=\left\{\left(z_{x}\right)_{x \in X}:\left(z_{x}\right)_{x \in X} \in K^{X}, \sup _{x \in X}\left|z_{x}\right|<\infty\right\}
\end{aligned}
$$

and

$$
\begin{aligned}
\psi(X)=\left\{\left(z_{x}\right)_{x \in X}:\left(z_{x}\right)_{x \in X}\right. & \in K^{X}, \\
& \left.\left(z_{x}\right)_{x \in X} \text { has finitely many non-zero coordinates }\right\} .
\end{aligned}
$$

For an arbitrary positive number $p$ with $I \leq p<\infty$, we usually give a norm on $l^{p}(X)$ and $\psi(X)$ such that

$$
\begin{aligned}
& \left\|\left(z_{x}\right)_{x \in \dot{X}}\right\|_{p}=\left(\sum_{x \in X}\left|z_{x}\right|^{p}\right)^{1 / p} \text { for }\left(z_{x}\right)_{x \in X} \in z^{p}(X), \\
& \left\|\left(z_{x}\right)_{x \in X}\right\|_{\infty}=\sup _{x \in X}\left|z_{x}\right| \text { for }\left(z_{x}\right)_{x \in X} \in \psi(X) .
\end{aligned}
$$

Then $\left(\imath^{p}(x),\|\|_{p}\right)$ is a Banach spece with the dual $2^{q}(x)$, where 
$1 / p+1 / q=1$ (if $p=1, q=\infty$ ). Then we have the following.

PROPOSITION 2. Let $p$ be a positive number with $1 \leq p \leq \infty$. Then $l^{p}(X)\left(\sigma\left(l^{p}(X), \psi(X)\right)\right)$ is a $\beta$-Montel space but not a Montel space.

Proof. As any $B\left(\psi(X), \ell^{p}(X)\right)$-bounded subset is finite dimensional, $l^{p}(X)\left(\sigma\left(l^{p}(X), \psi(X)\right)\right)$ is infra-barrelled for $1 \leq p \leq \infty$. Next we shall show that $Z^{p}(X)\left(\sigma\left(l^{p}(X), \psi(X)\right)\right)$ is $\beta$-semi-Montel but not semi-Montel.

For each $p$ with $1<p \leq \infty$, there is a positive $q$ such that $1 / p+1 / q=1$ (if $p=\infty, q=1$ ). $\left(\psi(X),\|\|_{q}\right)$ is a dense subspace of $\left(\imath^{q}(X),\|\|_{q}\right)$ with the dual $\imath^{p}(X)$. In case of $p=1,\left(\psi(X),\|\|_{\infty}\right)$ is a normed space with the dual $Z^{l}(X)$. Hence $Z^{p}(X)\left(\sigma\left(\tau^{p}(X), \psi(X)\right)\right\}$ is $\beta$-semi-Montel. On the other hand let $Y$ be a countable subset of $X$ such that $Y=\left\{x_{i}: x_{i} \in X, i \in N\right\}$ and we consider the sequence $\left\{2^{n}\right\}$ such that $z_{x}^{n}=i$ for $x=x_{i}, i=1,2, \ldots, n$ and $z_{x}^{n}=0$ for $x \neq x_{i}$, $i=1,2, \ldots, n$ for each $n \in N$. Then in $K^{X}\left(\sigma\left(K^{X}, \psi(X)\right)\right),\left\{z^{n}\right\}$ converges to $z=\left(z_{x}\right)_{x \in X}$ where $z_{x}=i$ for $x=x_{i}, i=1,2, \ldots$, and $Z_{x}=0$ otherwise. However $z$ does not belong to $2^{p}(X)$ for $1 \leq p \leq \infty$.

Consequently $Z^{p}(X)\left(\sigma\left(\tau^{p}(X), \psi(X)\right)\right)$ is not semi-Montel. This completes the proof.

\section{Some properties of $\beta-($ semi-)Montel spaces}

First of all we give a few permanence properties of $\beta$-(semi-) Montel spaces.

PROPOSITION 3. The product space $E(\tau)=\prod_{\alpha \in I} E_{\alpha}\left(\tau_{\alpha}\right)$ of $\beta$-(semi-) Montel spaces $E_{\alpha}\left(\tau_{\alpha}\right) \quad(\alpha \in I)$ is $B$-(semi-)Montel.

Proof. If $E_{\alpha}\left(\tau_{\alpha}\right) \quad(\alpha \in I)$ is infra-barrelled, $\prod_{\alpha \in I} E_{\alpha}\left(\tau_{\alpha}\right)$ is infra-barrelled. Let $E_{\alpha}^{\prime}(\alpha \in I)$ be the dual of $E_{\alpha}\left(\tau_{\alpha}\right)$ and 
$E_{\alpha}^{\prime}=\bigoplus_{\alpha \in I} E_{\alpha}^{\prime}$ be the dual of $E(\tau)$. As $E\left(\beta\left(E, E^{\prime}\right)\right)=\prod_{\alpha \in I} E_{\alpha}\left(\beta\left(E_{\alpha}, E_{\alpha}^{\prime}\right)\right)$ in the dual pair $\left(\prod_{\alpha \in I} E_{\alpha}, \underset{\alpha \in I}{\oplus} E_{\alpha}^{\prime}\right)$, for any $B\left(E, E^{\prime}\right)$-bounded subset $B$, there exists a $B_{\alpha}(\alpha \in I)$ which is $B\left(E_{\alpha}, E_{\alpha}^{\prime}\right)$-bounded and $B$ is contained in $\prod_{\alpha \in I} B_{\alpha}$. As each $B_{\alpha}$ is relatively $\tau_{\alpha}$-compact, $\prod_{\alpha \in I} B_{\alpha}$ is relatively compact in $E(\tau)$, so is $B$.

PROPOSITION 4. The locally convex direct sum $E(\tau)=\underset{\alpha \in I}{\oplus} E_{\alpha}\left(\tau_{\alpha}\right)$ of B-(sem-)Montel spaces $E_{\alpha}\left(\tau_{\alpha}\right)(\alpha \in I)$ is $\beta$-(semi-) Montel.

Proof. Let $E_{\alpha}^{\prime}(\alpha \in I)$ be the dual of $E_{\alpha}\left(\tau_{\alpha}\right)$ and $E^{\prime}=\prod_{\alpha \in I} E_{\alpha}^{\prime}$ be the dual of $E(\tau)$. As $E\left(\beta\left(E, E^{\prime}\right)\right)=\underset{\alpha \in I}{\oplus} E_{\alpha}\left(\beta\left(E_{\alpha}, E_{\alpha}^{\prime}\right)\right)$ in the dual pair $\left(\oplus_{\alpha \in I} E_{\alpha}, \prod_{\alpha \in I} E_{\alpha}^{\prime}\right)$, every $B\left(E, E^{\prime}\right)$-bounded subset $B$ is contained in $B_{\alpha_{1}}+\ldots+B_{\alpha_{n}}$ (each $B_{\alpha_{i}}$ is $\beta\left(E_{\alpha_{i}}, E_{\alpha_{i}^{\prime}}^{\prime}\right)$-bounded in $E_{\alpha_{i}}\left(\tau_{\alpha_{i}}\right)$, $i=1,2, \ldots, n)$. As $B_{\alpha_{1}}+\ldots+B_{\alpha_{n}}$ is relatively compact in $E(\tau)$, so is $B$. Clearly $E(\tau)$ is infra-barrelled if $E_{\alpha}\left(\tau_{\alpha}\right)(\alpha \in I)$ is infra-barrelled.

PROPOSITION 5. A closed subspace $H(\bar{\tau})$ of a B-semi-Montel space $E(\tau)$ is B-semi-Montel.

Proof. Let $H^{\prime}$ be the dual of $H(\bar{\tau})$ and $E^{\prime}$ be the dual of $E(\tau)$.

If $B$ is an arbitrary $B\left(H, H^{\prime}\right)$-bounded subset, it is $B\left(E, E^{\prime}\right)-$ bounded in $E(\tau)$ since $\beta\left(H, H^{\prime}\right)$ is finer than the topology $B\left(E, E^{\prime}\right)$ on H.

Hence $B$ is $\bar{\tau}$-relatively compact.

COROLLARY. A topological projective limit $E(\tau)=\lim A_{\alpha \beta}\left(E_{\beta}\left(\tau_{\beta}\right)\right)$ is B-semi-Montel if $E_{\alpha}\left(\tau_{\alpha}\right) \quad(\alpha \in I)$ is B-semi-Montel.

Next we give the other properties related to $\beta$-(semi-)Montel spaces.

PROPOSITION 6. Let $E(\tau)$ be a countably barrelled, separable and metrizable locally convex space and $E^{\prime}$ be its dual. If $N$ is a 
countable dense subset in $E(\tau)$ and $E$ is a subspace of $E$ which is spanned by $N$, then $E^{\prime}\left(\sigma\left(E^{\prime}, F\right)\right)$ is a B-Montel space.

Proof. $E^{\prime}\left(\sigma\left(E^{\prime}, F\right)\right)$ is metrizable since it has a countable base of o-neighbourhoods. $E(\tau)$ is barrelled from the assumption. If $B$ is any $\sigma\left(E^{\prime}, E\right)$-bounded subset, it is relatively $\sigma\left(E^{\prime}, E\right)$-compact. To show $\sigma\left(E^{\prime}, E\right) \leq \beta\left(E^{\prime}, F\right)$, it suffices to show that for each element $x$ of $E$, there is a $\sigma\left(F, E^{\prime}\right)$-bounded subset $C$ such that $x$ is an element of I-closure of $C$. For an arbitrary element $x$ of $E$, there is a sequence $\left\{x_{n}\right\}$ such that each $x_{n}$ is an element of $F$ and $\left\{x_{n}\right\}$ converges to $x$ from its separability and metrizability. If $C$ is the sequence $\left\{x_{n}\right\}$, it is $\sigma\left(F, E^{\prime}\right)$-bounded and $x$ is an element of $\tau$-closure of $C$. Now if $B$ is an arbitrary $B\left(E^{\prime}, F\right)$-bounded subset, it is $\sigma\left(E^{\prime}, E\right)$-bounded.

Thus $B$ is relatively $\sigma\left(E^{\prime}, F\right)$-compact. So $E^{\prime}\left(\sigma\left(E^{\prime}, F\right)\right)$ is B-Montel.

Let $E(\tau)$ be a Montel space and $E^{\prime}$ be its dual. Then the strong dual $E^{\prime}\left(B\left(E^{\prime}, E\right)\right)$ is also a Montel space. In case of a B-Montel space, a similar proposition holds.

PROPOSITION 7. Let $E(\tau)$ be a B-Montel space and $E^{\prime}$ be its dual. Then $E^{\prime}\left(\beta^{*}\left(E^{\prime}, E\right)\right)$ is B-Montel.

Proof. If $B$ is any absolutely convex and $B\left(E, E^{\prime}\right)$-bounded subset, it is relatively $\sigma\left(E, E^{\prime}\right)$-compact from the assumption. Then $E^{\prime}\left(\tau\left(E^{\prime}, E\right)\right)$ is infra-barrelled since $\beta^{*}\left(E^{\prime}, E\right)=\tau\left(E^{\prime}, E\right)$. Next we show that any $B\left(E^{\prime}, E\right)$-bounded subset in $E^{\prime}\left(\tau\left(E^{\prime}, E\right)\right)$ is relatively $\tau\left(E^{\prime}, E\right)$-compact.

If $B$ is any $B\left(E, E^{\prime}\right)$-bounded subset in $E(\tau)$, it is $\tau$-precompact. From this $\tau\left(E^{\prime}, E\right)=\beta^{*}\left(E^{\prime}, E\right) \leq \tau_{c}\left(E^{\prime}, E\right)$. If $C$ is an arbitrary $B\left(E^{\prime}, E\right)$-bounded subset in $E^{\prime}\left(\tau\left(E^{\prime}, E\right)\right)$, it is a $\tau$-equicontinuous subset.

Consequently $C$ is relatively $\sigma\left(E^{\prime}, E\right)$-compact and relatively $\tau_{c}\left(E^{\prime}, E\right)$-compact from the property of the topology $\tau_{c}\left(E^{\prime}, E\right)$. Hence it is relatively $\tau\left(E^{\prime}, E\right)$-compact. Thus $E^{\prime}\left(\beta^{*}\left(E^{\prime}, E\right)\right)$ is B-Montel.

Using Proposition 7, we can give a B-Montel space whose completion is not B-semi-Montel. 
EXAMPLE 4. From Example 3, $l^{l}\left(\sigma\left(l^{l}, \psi\right)\right)$ is B-Montel. $\psi\left(\beta^{*}\left(\psi, Z^{1}\right)\right)$ is $\beta$-Montel from Proposition 7 . On the other hand, $\left(\psi,\|\|_{\infty}\right)$ is a normed space with the dual $l^{\mathcal{L}}$. Thus $\left(\psi,\|\|_{\infty}\right)$ is B-Montel and the completion of $\left(\psi,\|\|_{\infty}\right)$ is $\left(c_{0},\|\|_{\infty}\right)$. As $\left(c_{0},\|\|_{\infty}\right)$ is an infinite dimensional Banach space, it is not $\beta$-semi-Montel.

From Example 4 we obtain the following.

THEOREM 1. Under the conditions of Proposition 6, the subspace $F(\bar{\tau})$ of $E(\tau)$ is a B-Montel space.

Proof. From Proposition 6, $E^{\prime}\left(\sigma\left(E^{\prime}, F\right)\right)$ is a $\beta$-Montel space and $F\left(\beta^{*}\left(F, E^{\prime}\right)\right)$ is a $\beta$-Montel space from Proposition $7 . \quad F(\bar{\tau})$ is a dense subspace of $E(\tau)$, so the dual of $F(\bar{\tau})$ is $E^{\prime}$.

As $F(\bar{\tau})$ is metrizable, $F(\bar{\tau})=F\left(\beta^{*}\left(F, E^{\prime}\right)\right)$.

REMARK 2. Let $E(\tau)$ be the strict inductive limit of $\beta$-semi-Montel spaces $E_{n}\left(\tau_{n}\right), n=1,2, \cdots$, and $E^{\prime}$ be the dual of $E(\tau)$ and $B$ be an arbitrary $\beta\left(E, E^{\prime}\right)$-bounded subset in $E(\tau)$. Then it is not known whether we can find a space $E_{n}\left(\tau_{n}\right)$ where $B$ is $\beta\left(E_{n}, E_{n}^{\prime}\right)$-bounded. ( $E_{n}^{\prime}$ is the dual of $E_{n}\left(\tau_{n}\right)$.) About the other construction appeared in [7], as in the case of a (semi-) Montel space, a $\beta$-(semi-) Montel space is not always preserved under them.

\section{Separability of a metrizable B-Montel space}

In general, every Fréchet Montel space is separable. In this section we consider whether every metrizable B-Montel space is separable. In fact the space is not always separable. Here we give an example of the above fact.

EXAMPLE 5. Let $X$ be a set such that $|X|>\aleph_{0}$. From Proposition 2, $l^{\perp}(X)\left(\sigma\left(l^{l}(X), \psi(X)\right)\right)$ is B-Montel; and from Proposition 7 , $\psi(X)\left(\beta^{*}\left(\psi(X), 2^{1}(X)\right\}\right)$ is also $\beta$-Montel. However $\left(\psi(X),\|\|_{\infty}\right)$ is a normed space whose dual is $l^{1}(X)$. Hence $\left(\psi(X),\|\|_{\infty}\right)$ is a metrizable B-Montel space and clearly not separable. 
However we obtain a theorem that a metrizable B-Montel space is separable under the following condition.

THEOREM 2. Let $E(\tau)$ be a metrizable $\beta$-Montel space and $E^{\prime}$ be its dual. If $E^{\prime}\left(\sigma\left(E^{\prime}, E\right)\right)$ has a fundamental sequence of bounded subsets, $E(\tau)$ is separable.

Proof. $E\left(B\left(E, E^{\prime}\right)\right)$ is metrizable from the assumption. Let \|\|$_{\tau}$ and \|\|$_{\beta}$ be the $F$-norms on $E(\tau)$ and $E\left(B\left(E, E^{\prime}\right)\right)$. Then if $E(\tau)$ is not separable, there exist a positive number $\delta$ and an uncountable subset $A$ of $E$ such that $\left\|z-z^{\prime}\right\|_{\tau}>\delta$, for $z, z^{\prime} \in A, z \neq z^{\prime}$.

For $n \in N$ we put $K_{n}=\left\{x:\|x\|_{B}<1 / n, x \in E\right\}$ and for an arbitrary $x \in E$, we denote $\inf \left\{t: t>0, x / t \in K_{i n}\right\}$ by $[x]_{n}$. Then there is a positive number $M_{1}$ such that $A_{1}=A \cap\left\{x:[x]_{1}<M_{1}, x \in E\right\}$ is an uncountable set.

Similarly for each $n \in N, n \geq 2$, there is a positive number $M_{n}$ such that $A_{n}=A_{n-1} \cap\left\{x:[x]_{n}<M_{n}, x \in E\right\}$ is an uncountable set. We obtain a sequence of uncountable subsets of $E$. For each $A_{j}$ we take an element $z_{j} \in A_{j} \cdot\left\{z_{j}\right\}$ is $B\left(E, E^{\prime}\right)$-bounded since for each $K_{n}$, $\left\{z_{j}\right\} \subset \max \left\{m_{1}, m_{2}, \ldots, m_{n-1}, M_{n}\right\} \cdot K_{n}\left(z_{i} \in m_{i} \cdot K_{n}, i=1,2, \ldots, n-1\right)$. Hence $\left\{z_{j}\right\}$ is relatively $\tau$-compact. On the other hand, $\left\|z_{i}-z_{j}\right\|_{\tau}>\delta$ for $i \neq j$. This leads to a contradiction.

REMARK 3. As an example of Theorem $2,{ }_{2}^{p}\left(\sigma\left(2^{p}, \psi\right)\right)(1 \leq p \leq \infty)$ is given. Conversely let $E(\tau)$ be a separable, metrizable and B-Montel space and $E^{\prime}$ be its dual. Then $E^{\prime}\left(\sigma\left(E^{\prime}, E\right)\right)$ does not always have a fundamental sequence of bounded subsets. For example, we put $E(\tau)=\left(\psi,\|\|_{\infty}\right)$ then the weak dual $\tau^{\perp}\left(\sigma\left(z^{\perp}, \psi\right)\right)$ does not have a fundamental sequence of bounded subsets. 


\section{References}

[1] N. Adasch, B. Ernst and D. Keim, Topological vector spaces (SpringerVerlag, Berlin, Heidelberg, New York, 1978).

[2] D. Gulick, "Duality theory for the topology of simple convergence", $J$. Math. Pures Appl. 52 (1973), 453-472.

[3] J. Hórvath, Topological vector spaces and distributions, Vol. I

(Addison-Wesley, Reading, Massachusetts, 1966).

[4] T. Husain, "Two new classes of locally convex spaces", Math. Ann. 166 (1966), 289-299.

[5] P.K. Kamthan and M. Gupta, Sequences spaces and series (Lecture Notes 65. Marcel Dekker, New York, 1981).

[6] G. Köthe, Topological vector spaces 1 (translated by D.J. Garling.

Die Grundlehren der mathematischen Wissenschaften, 159.

Springer-Verlag, Berlin, Heidelberg, New York, 1969).

[7] K. Mckennon and J.M. Robertson, Locally convex spaces (Marcel Dekker, New York and Basel, 1976).

Department of Mathematics,

Kobe University,

Nada-ku,

Kobe 657,

Japan. 